\title{
Nucleic acid approaches for detection and identification of biological warfare and infectious disease agents
}

\author{
Dmitri Ivnitski ${ }^{1}$, Daniel J. O’Neil ${ }^{2}$, Anthony Gattuso ${ }^{3}$, Roger Schlicht $^{3}$, \\ Michael Calidonna ${ }^{4}$, and Rodney Fisher ${ }^{4}$
}

BioTechniques 35:862-869(October 2003)

\begin{abstract}
Biological warfare agents are the most problematic of the weapons of mass destruction and terror. Both civilian and military sources predict that over the next decade the threat from proliferation of these agents will increase significantly. In this review we summarize the state of the art in detection and identification of biological threat agents based on PCR technology with emphasis on the new technology of microarrays. The advantages and limitations of real-time PCR technology and a review of the literature as it applies to pathogen and virus detection are presented. The paper covers a number of issues related to the challenges facing biological threat agent detection technologies and identifies critical components that must be overcome for the emergence of reliable PCR-based DNA technologies as bioterrorism countermeasures and for environmental applications. The review evaluates various system components developed for an integrated DNA microchip and the potential applications of the next generation of fully automated DNA analyzers with integrated sample preparation and biosensing elements. The article also reviews promising devices and technologies that are near to being, or have been, commercialized.
\end{abstract}

\section{INTRODUCTION}

Biological warfare agents (BWAs) in the form of bacteria, viruses, fungi, and toxins are the most problematic of the weapons of mass destruction (1-5). BWA aerosols are usually invisible, odor- and taste-free, and difficult to detect after the initial release. Unlike an attack with a chemical agent, an attack with a BWA usually does not cause an immediate reaction. An incubation period of up to several days is often required before a victim displays serious symptoms characteristic of having been exposed to a biological pathogen. Consequently, the effect such an attack has on individual victims and the rate of spread is masked and magnified before detection.

Because of the clandestine nature of dispersal and the potential lethality of many agents, BWAs could kill more people than a nuclear or chemical attack. For example, 10 grams of weaponized anthrax spores could result in the deaths of as many people as an attack using a ton of the nerve agent sarin. Compounding the threat is the fact that many BWAs are communicable, and thus may be readily passed from person to person. Moreover, the potential threat has been magnified in recent years by advances in molecular biology, genetic engineering, and related technologies, as well as in the development of more efficient delivery and dispersion systems. Therefore, the ability to accurately predict the dispersion, concentration, and ultimate fate of BWAs released into the environment in real time is essential to prepare for and respond to a BWA release. Upon and during attack by BWAs, biodetectors preferably should be decentralized and networked to allow the definition of the perimeter of attack and moving fronts, while also having the capability of being moved into the field or area of attack where rapid diagnosis and monitoring can be undertaken. Ideally, a response network should incorporate remote sensing devices and point detectors. However, development of networked biodetectors is a very complicated task because thousands of different pathogenic microorganisms could be used in an attack. Another problem is a huge background bio-aerosol, the myriad similar nonpathogenic microorganisms constantly present in the environment. Because many pathogenic organisms differ little from normal flora, a practical detection system has to discriminate between closely related organisms.

Overall, this situation has created a rapidly rising demand for new emerging sensor technologies to speed up testing. The future of emerging biosensor technology is the ability to create network systems based on a combination of reliable and portable alarm-type biodetectors and more sophisticated

\footnotetext{
${ }^{1}$ New Mexico Institute of Mining and Technology, Socorro, NM, ${ }^{2}$ CRADA International, Atlanta, GA,

${ }^{3}$ General Atomics, San Diego, CA, and ${ }^{4}$ Air Force Research Laboratory, Tyndall Air Force Base, FL, USA
} 
detectors/identifiers. The alarm-type detectors should provide generic discrimination of environmental species (e.g., biological vs. nonbiological species or pathogenic vs. nonpathogenic agents), and they may be used as "triggers" (i.e., precursors and complements) for a more sophisticated detector/identifier system. New biodetectors should be capable of being used anytime and anywhere and should take advantage of remote, wireless communications, such as those used successfully in environmental monitoring and reporting and in telemedicine applications (2-5). The effective testing of biological agents requires portable sensor technology, which should be extremely sensitive, universal, reliable, and fast (Figure 1). It should be miniaturized, use fewer consumables (to minimize a logistics footprint), and be of low maintenance relative to current equipment that has been deployed for real-time monitoring of BWAs. A sensor should be able to detect biological agents at threshold concentrations in a minimum of 5-10 min. There is also a growing need for portable, highly sensitive, and fast biodetectors capable of detecting and identifying bio-threat agents in the field. Portability, rather than transportability, requires lighter weights, smaller volumes, moderate power consumption, and enhanced energy efficiency.

Unfortunately, current biological detection systems have not kept pace with the threat posed by BWAs. Commercially available chemical detection systems are much more robust and developed than counterpart systems to detect BWAs (4-6). Chemical detectors can provide information about chemical warfare agents within seconds or minutes, under field conditions. At the same time, very few biodetectors for BWAs suitable for use under field conditions are commercially available. Conventional biodetection systems have several shortcomings that must be overcome: (i) they are slow to recognize the presence of a pathogen; (ii) they are not suitable to discriminate simultaneously a full set of pathogenic versus nonpathogenic microorganisms in the environment; (iii) except in laboratory settings, they lack adequate sensitivity; (iv) the systems are often transportable rather than portable and are cumbersome for field operations; $(v)$ they require highly

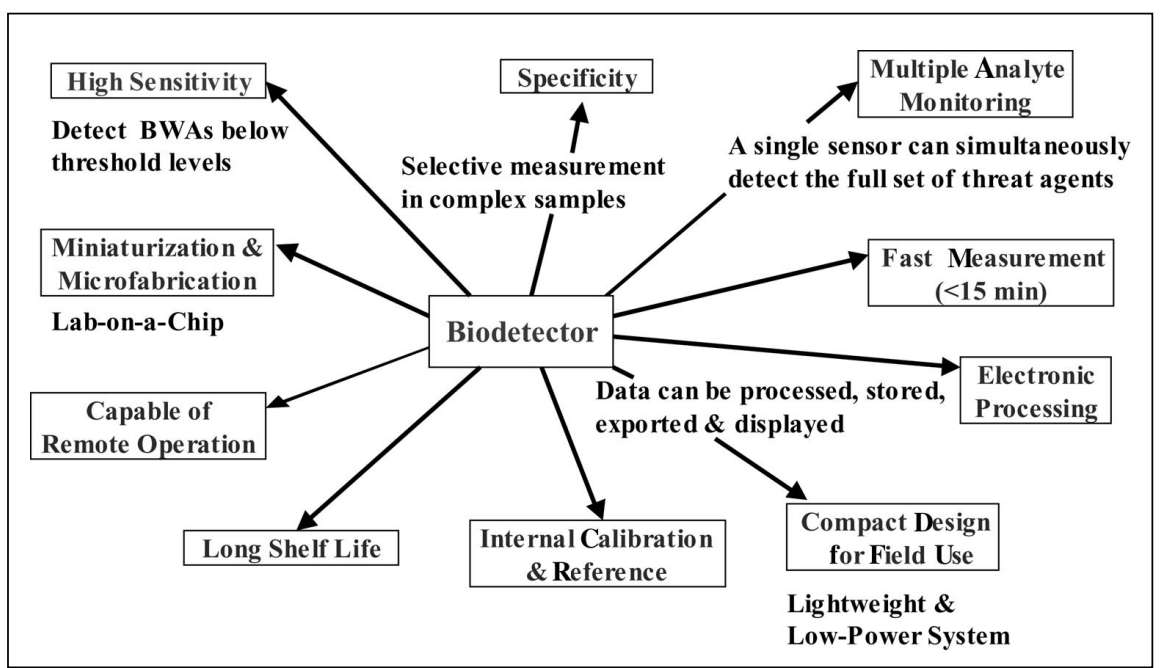

Figure 1. Main requirements for biodetector systems for detection and identification of biological warfare agents. trained personnel to properly operate them; (vi) they cannot be monitored or operated by remote control; and (vii) their purchase, maintenance, and operation are expensive. Other limitations are the complexity of the instrumentation, multistep assay processes, and the time-consuming procedures that are always required.

Recent advances in areas such as microarray technology, microelectromechanical systems, microfluidics, and optoelectronics present new technological possibilities for producing fast, extremely sensitive, and inexpensive "smart" sensing systems for field application (7-10). In this review we examine the current state of the art in detection and identification of BWAs based on approaches using nucleic acids, focusing primarily on PCR technology and the newly emerging technology of microarrays. The advantages and limitations of real-time PCR technology and a review of the literature as it applies to pathogen and virus detection are presented. While promising, there are a number of significant challenges involved with PCR and microarray detection of BWAs that must be overcome for the emergence of reliable systems that can serve as gold standards.

\section{PCR-BASED TECHNOLOGIES}

Rapid and accurate identification of the full set of BWAs in the environment is a key element of biological defense strategy (3-19). To date, detection and identification strategies based on real-time PCR technology have found the greatest use for BWA analysis because of its rapidity, sensitivity, and reproducibility, and the reduced risk of human error. Significant recent advances in PCR chemistry and thermal cycling technology have compressed the timeframe for analysis from several hours to a few minutes (18-27).

Nucleic acid-based diagnostic assays demand detection of organisms or DNA mutations at very low concentrations, often less than 100 copies per milliliter, in raw biological samples such as blood or urine. Such sensitivity requirements mandate the use of minimum quantities of the starting sample. PCR is capable of detecting fewer than 20 copies of a target in any given biological sample. If the required assay sensitivity is 100 copies per milliliter, then a minimum of 200 $\mu \mathrm{L}$ of the original sample must be processed to ensure statistical confidence in the result of the assay. Advanced fluidic processing technologies that bridge the gap between the demand for "large" sample volumes and the world of microstructures and microarrays have been reported $(28,29)$.

Cepheid (Sunnyvale, CA, USA) has developed two approaches to bridging this gap $(28,29)$. In one approach, they use their ${\mathrm{I}-C O R E^{\circledR}}^{\circledR}$ (intelligent cooling/heating optical reaction) technology, a thermal-cycling technology for performing fast PCR on large sample 
volumes. In a second approach, they address the problem of efficiently miniaturizing the next "upstream" fluid processes in nucleic acid assays: nucleic acid purification and concentration. In this context, Cepheid has developed a micromachined, microfluidic technology in which high-surface-area structures are created in silicon substrates. Such structures are designed into well-defined arrays that optimize fluid flow and liquid-surface interaction. Although the internal volume of such a chip is several microliters, its array of micromachined columns can capture dissolved nucleic acid as it flows through the chip. Cepheid's fluidic cartridges are designed to perform the following functions: (i) reagent containment and delivery; (ii) sample and reagent mixing; (iii) cell separation and concentration using modular filter and solid-phase holder assemblies; (iv) rapid cell lysis using ultrasonic techniques; (v) DNA or RNA capture, enrichment, and purification using chips or solid-phase materials; and (vi) preparation of reaction mixture and filling integrated PCR tubes. The fluidic cartridges are used for the I-CORE modules. Each I-CORE module includes a four-channel optical analysis system capable of detecting and quantifying multiple fluorescent dyes and multiple target molecules in the same reaction tube. I-CORE technology can achieve less than 30-s PCR cycles for volumes as large as $100 \mu \mathrm{L}$, while simultaneously detecting and quantifying fluorescent DNA products in real time. The steps associated with DNA extraction, amplification, and detection are fully integrated in one system and, therefore, in one procedure. Based on the I-CORE module, the company developed a family of DNA analysis instruments including the Microfluidic Integrated DNA Analysis System $\left(\right.$ MIDAS $\left.^{\circledR}\right)$ and Smart Cycler ${ }^{\circledR}$ DNA detection systems. The MIDAS can identify BWAs in less than $30 \mathrm{~min}$. Along with unprecedented processing speed, MIDAS is totally automated, requiring the operator only to inject a specimen into the machine. The commercial successor to MIDAS, called GeneXpert ${ }^{\circledR}$, uses the same microfluidics technology integrated into disposable assay cartridges. The cartridges contain all the specific reagents required to detect disease organisms such as Bacillus anthracis, Chlamydia trachomatis, or foodborne pathogens. MIDAS performs all the complicated handling of reagents and samples, amplifies the DNA, and detects specific sequences of viruses, bacteria, or spores. The system then cleans and decontaminates itself and is ready for another assay.

Recently, the Centers for Disease Control and Prevention (CDC, Atlanta, GA, USA) developed and validated test kits for several biological threat agents for use on the Smart Cycler DNA detection systems. The Smart Cycler is designed for both laboratory and field application. The Smart Cycler II TD system is a ruggedized model, for field testing including a theater of operations during warfare (30). The Smart Cycler II is built with 16 I-CORE modules and enables rapid sample analysis by real-time PCR with results in as little as $20 \mathrm{~min}$.

Another strategy for rapid detection and identification of bacteria has been proposed by Applied Biosystems (Foster City, CA, USA) (31). The company, a part of Applera (Norwalk, CT, USA; formerly Perkin Elmer) has developed a new homogeneous fluorescence bead-based immunoassay, the FLISA, which requires no wash and only one incubation step (32). The FLISA (fluorescence-linked immunosorbent assay) uses $6-\mu \mathrm{m}$ antibody-coated polystyrene beads. The immunoassays were developed for the cytokines IL-6 and IL-8. The FLISA is comparable to traditional ELISA with respect to linear dynamic range and sensitivity and can be readily performed in 96- and 384-well plates. Additionally, the FLISA utilizes 100 times less primary antibody than the conventional immunoassay. While scanning, the laser is focused on the bottom of the well, and the fluorescence associated with each bead is detected over the unbound, background fluorescence. An additional key feature of the FLISA system is its multiplex capability using different bead sizes and dyes. The multiplexed system can detect different PCR products simultaneously $(31,32)$. Recently the company developed a 7900HT Immune Profiling Micro Fluidic card that can analyze 384 TaqMan $^{\circledR}$-based assays simultaneously on a single card.

While the speed and sensitivity of nucleic acid analysis based on PCR has been significantly improved, the PCR technique requires that the bacteria and spores be disrupted to make the endogenous DNA available for amplification. Bacterial spores are particularly difficult to process, as their nucleic acid is encased in a very resistant shell. Spore lysis methods have included chemical, enzymatic, mechanical, and thermal treatments (33-35). However, physical disruption methods are preferred, as most chemical agents inhibit the PCR process, requiring removal in subsequent additional steps. Researchers at Lawrence Livermore National Laboratory have developed a minisonicator and spore lysis cartridge that allow the disruption of bacillus spores in $30 \mathrm{~s} \mathrm{(35).} \mathrm{Uti-}$ lization of the minisonicator significantly improved PCR analysis by decreasing the limit of detection and the time of assay. The total time of spore disruption and detection using the minisonicator and the Advanced Nucleic Acid Analyzer (ANAA) was less than 15 min. The ANAA contains an array of 10 thermo-optic modules, improved software, and a modified detection system $(30,35-38)$. The ANAA, with its laptop computer, performs a full PCR analysis of a normal-size cellular culture in approximately $7 \mathrm{~min}$. A full PCR analysis includes cell lysis, PCR, detection of the PCR product with a target-specific fluorescence resonance energy transfer probe, and automated alerting of a positive signal. A sample is introduced into a $25-\mu \mathrm{L}$ plastic tube, inserted into the siliconchip microchamber along with several chemicals, including the polymerase enzyme, PCR buffers, nucleic-acid primers, and a fluorogenic nucleic-acid probe. The sample with the reagents is first heated to just below boiling $\left(96^{\circ} \mathrm{C}\right)$ and then cooled to $56^{\circ} \mathrm{C}$. This cycle is repeated. During each cycle of the temperature, PCR replicates the target sequence of DNA, eventually resulting in billions of strands of this DNA for testing. In addition, the chip has an integrated thin-film heating element for controlling the chamber's temperature, a blue light-emitting diode (LED) to excite the chemical probe, and two photodiodes with 530- and 590-nm bandpass filters, respectively, for detecting the resulting fluorescent signals.

Lawrence Livermore National Laboratory scientists have also developed a four-chamber, battery powered, handheld instrument referred to as the Handheld Advanced Nucleic Acid Analyzer (HANAA). The HANAA is an instrument capable 
of rapid detection and identification of biowarfare and bioterrorism agents in the field using a TaqMan ${ }^{\circledR}$-based PCR assay. It is a highly automated device, able to automatically prepare samples, then simultaneously test up to four different samples for two different DNA sequences each, and report the results in about $30 \mathrm{~min}$. The HANAA system could, in principle, detect as few as 10 individual bacteria including $B$. anthracis . About the size of a brick, the HANAA biodetection system can be held in one hand and weighs less than a kilogram. The system was designed for emergency response groups, such as firefighters and police, who are often first on the scene at sites where bioterrorism may have occurred.

Another automated PCR-based system is the Ruggedized Advanced Pathogen Identification Device (R.A.P.I.D. ${ }^{\circledR}$; Idaho Technology, Salt Lake City, UT, USA), which is based on the Light-Cycler ${ }^{\circledR}$ PCR technology with a fluorescence detection system $(13,18,39-41)$. Developed originally for military field hospitals and other rough environments, the R.A.P.I.D. is a field-deployable rapid thermal cycler with fluorescence monitoring. The principle of PCR-monitoring with the LightCycler System is based on hybridization probes. The two probes are designed to hybridize to the specific target in a head-to-tail arrangement that brings the two different dyes (fluorophore) into close proximity to each other, which in turn leads to the transfer of fluorescent resonance energy. Hybridization probes could have an advantage over hydrolysis and hairpin probes because they are labeled with only a single dye. The R.A.P.I.D. is capable of automatically analyzing samples for the presence of any nucleic acid sequences. Up to 32 prepared test samples may be amplified and analyzed within $40 \mathrm{~min}$. Up to 10 different organisms may be tested for in a single screen test. The basic instrument weighs less than $25 \mathrm{~kg}$ and is designed to be air, land, and sea transportable. An advanced artificial intelligence system allows the R.A.P.I.D. to automatically collect the data, interpret the test data, and report the results, providing the capability for pathogen testing and identification in the field.

The advantages of the Idaho Technology's original LightCycler attracted attention and investment from Roche Molecular Biochemicals. Roche's cornerstone system, the COBAS AMPLICOR ${ }^{\mathrm{TM}}$ Analyzer, launched in Europe in 1995 and in the United States in 1997, was the first system to automate both the amplification and detection steps of the PCR testing process by combining five instruments into one (thermal cycler, automatic pipettor, incubator, washer, and reader) for the detection and quantification of different infectious agents including detection of $B$. anthracis DNA $(14,18,42,43)$. By first using the Roche COBAS AmpliPrep ${ }^{\mathrm{TM}}$ System to prepare PCR specimens and then employing the COBAS TaqMan System, which automates the amplification, detection, and quantification of DNA or RNA, PCR analysis is fully automated. The system contains a single thermal cycler with two independently regulated heating/cooling blocks, an incubator, a magnetic particle washer, a pipettor, and a photometer. Amplified products are captured on oligonucleotidecoated paramagnetic microparticles and detected with use of an avidin-horseradish peroxidase (HRP) conjugate. To avoid DNA carryover, separate dedicated areas are used for reagent preparation, sample preparation, and detection. No cross-re- activity was observed when three different target nucleic acids were amplified in a single reaction and detected with three target-specific capture probes. There are now close to 4000 COBAS AMPLICOR systems in clinical settings worldwide. The system has a broad test menu with the ability to test for B. anthracis, C. trachomatis, Neiserria gonorrhea, Mycobacterium tuberculosis, cytomegalovirus, hepatitis B virus, hepatitis $\mathrm{C}$ virus, and human imunodeficiency viruses.

\section{MICROARRAY TECHNOLOGIES}

Miniaturization of biodetectors into a single, integrated, "lab-on-a-chip" system offers great potential for environmental monitoring, which includes improved accuracy, lower power and sample consumption, disposability, and automation (44-51). This technology adapts microfabrication techniques used in semiconductor manufacture to convert experimental and analytical protocols into chip architectures. Already chips are being fabricated with picoliter-size wells and $10-\mu \mathrm{L}$-size chambers for sample preparation and detection (51-61). The integration of microfluidic transport, total automation, and materials handling contributes to a major reduction in system retention and material transfer losses, which increases accuracy and reduces sample size requirements. However, one of the remaining barriers to achieving true miniaturized total analysis systems is the integration of sample pretreatment for microfluidic devices. The challenge is complicated by the complexity and variation in prospective samples and analytes. There is an issue of integration and interfacing the pretreatment operation to the analysis device with which it is coupled and codependent in terms of sample volume, time, and reagent and power consumption $(62,63)$. The majority of published work has concentrated on using electrokinetically driven separation schemes to separate and detect analytes of interest (64-67). The electrokinetic phenomenon occurs due to the interaction of induced dipole in the bioparticles with electric fields and is used for movement of fluids and other materials through a network of fluid channels. In this case, external pumps or valves are not needed. Precise control of fluid motion and reaction timing is achieved by changing parameters such as the current or voltage. The chip-based capillary electrophoresis system has the capacity to perform the following functions: reagent dispensing, mixing, incubation, reaction, and sample partition and analyte detection. Evidence in the experimental data produced by different organizations has shown that microchip electrophoresis is an effective process for analyzing biological agents at very high speeds and low concentrations (64-67). Chip-based capillary electrophoresis technology has many benefits if compared to conventional methods of analysis. For example, the chip can analyze a mixture in seconds where it would take capillary electrophoresis at least $20 \mathrm{~min}$ and gel electrophoresis $1 \mathrm{~h}$ to do the same analysis. The microchip can detect a sample concentration in the range of $100 \mathrm{pM}$, which is at least two orders of magnitude greater than conventional capillary electrophoretic analysis. PCR amplification of single DNA template molecules, followed by capillary electrophoretic analysis of the products, has been demonstrated in an integrated micro- 
fluidic device (66). The microdevice consists of submicroliter PCR chambers, etched into a glass substrate, which are directly connected to a microfabricated capillary electrophoresis system. Valves and hydrophobic vents provide controlled and sensorless loading of the 280-nL PCR chambers, low volume reactor, and low thermal mass. The use of thin-film heaters permits cycle times as fast as $30 \mathrm{~s}$. In operation, an amplified product, labeled with an intercalating fluorescent dye, is directly injected into a gel-filled capillary channel for electrophoretic analysis. This microchip electrophoresis has proved to be quicker, more sensitive, and cheaper than conventional techniques.

Several systems that use such chip-based or microarray technologies are commercially available. For example, ACLARA BioSciences (Mountain View, CA, USA) has developed integrated, automated, microfluidic systems in which a number of sample preparation and analytical steps, such as mixing, reaction, and separation, are performed on a singleuse plastic LabCard ${ }^{\circledR}$ (52). The focus of Caliper Technologies (Mountain View, CA, USA) is to develop microfluidic laboratory-on-a-chip technologies and products, marketed as LabChip $^{\mathrm{TM}}$ technologies $(53,54)$.

Affymetrix (Santa Clara, CA, USA) produces the GeneChip ${ }^{\circledR}$ probe arrays, which are manufactured by bonding hundreds of genetic sequences onto the surface of a microchip using photolithographic processes such as photosensitive masks, chemical doping layers, and other techniques used in computer chip fabrication (55-57). In October 2002, Affymetrix publicly announced the commercial availability of a new GeneChip ${ }^{\circledR}$ brand CustomSeq ${ }^{\mathrm{TM}}$ Resequencing Array that can sequence 30,000 bases in 2 days at a claimed accuracy of $99.99 \%$. Applications include pathogen subtyping to identify known and novel strains of microorganisms.

Genometrix (The Woodlands, TX, USA), acquired in 2002 by High Throughput Genomics (Tucson, AZ, USA), has developed several platforms that find applications in geneexpression analysis, mutation screening, and drug discovery (58). The company focused its attention in the direction of developing low-cost, medium-density tools that have the potential to screen a large number of samples (tens of thousands of tissues or different drug compounds) in parallel. The company developed an automated workstation for sample processing and automated chip hybridization. The workstation allows mRNA isolation, automated PCR, hybridization, and chip detection at the rate of up to 1000 samples per day.

Agilent's (Palo Alto, CA, USA) 2100 Bioanalyzer is another promising system for real-time analysis of different BWAs (59-61). Their approach is based on the integration of three main technologies: DNA microarrays, bioinformatics (using the Rosetta Resolver ${ }^{\circledR}$ gene expression data-analysis system), and lab-on-a-chip automated bioanalyzer product line (59). The system was developed collaboratively with Caliper Technologies. Using proprietary microfluidic breakthroughs from Caliper, the BioAnalyzer integrates fluid handling, sample processing, separation, and detection in a miniature chip format. A linear range for quantitative analysis is from 0.5 to $50 \mathrm{ng} / \mu \mathrm{L}$.

Gene Logic offers the Flow-Thru Chip ${ }^{\mathrm{TM}}$ (FTC) in which molecular interaction occurs within the 3-D volumes of po- rous substrates $(44,68,69)$. The FTC is a uniformly porous glass or silicon wafer, in contrast to flat, impenetrable materials used in traditional microarrays. In FTC technology, molecular interactions occur within the 3-D volumes of ordered microchannels rather than on the 2-D flat surface platforms available in the current market. The large surface areas of the microchannels allow an increase in both the sensitivity and spatial resolution required for quantitative chemiluminescence measurements on microarrays.

\section{HYBRID TECHNOLOGIES}

Scientists at Lawrence Livermore National Laboratory have also invented a stand-alone system for rapid, continuous monitoring of multiple airborne biological threat agents in the environment. This system, the autonomous pathogen detection system (APDS), acts as a biological "smoke alarm" and is targeted for domestic applications in which the public is at high risk of exposure to covert releases of bioagent (such as mass transit, office complexes, and convention centers), and as part of a monitoring network for urban areas and major gatherings. The APDS is completely automated, offering aerosol sampling, in-line sample preparation fluidics, multiplex flow cytometer detection and identification assays, and orthogonal, flow-through PCR (nucleic acid) amplification and detection. For the flow-cytometry subsystem, small "capture" beads $5 \mu \mathrm{m}$ in diameter are coated with antibodies specific to the target pathogens. The beads are color-coded according to which antibodies they hold. Once the pathogens attach to their respective antibodies, more antibodies (labeled with a fluorescent dye), are added to the mixture. A labeled antibody will stick to its respective pathogen, creating a sort of bead sandwich — antibody, pathogen, and labeled antibody. The beads flow one by one through a flow cytometer, which illuminates each bead in turn with a laser beam. Any bead with labeled antibodies will fluoresce. The system can then identify which agents are present, depending on the color of the capture bead, and has several key advantages over competing technologies: (i) the ability to measure up to 100 different agents and controls in a single sample; (ii) the flexibility and ease with which new bead-based assays can be developed and integrated into the system; (iii) low false-positive and false-negative detection due to the presence of two orthogonal detection methods; (iv) the ability to use the same basic system components for multiple deployment architectures; and (v) the relatively low cost per assay and minimal consumables.

Nanogen (San Diego, CA, USA) has focused on DNA diagnostics using a combination of electrophoresis, long DNA probes, and imaging techniques $(70,71)$. This technology comprises an electronically addressable silicon microchip that can be loaded with DNA capture probes using electrophoretic forces to bind the probes to specific sites on a chip array. They are refining their system to isolate and detect biological warfare and infectious-disease agents in clinical specimens.

Motorola Life Science (Pasadena, CA, USA) is developing its eSensor ${ }^{\mathrm{TM}}$ DNA Detection System, which examines up to 36 DNA or RNA targets simultaneously by bioelectron- 
ic detection, enabling convenient, flexible, and cost-effective tests $(72,73)$. The bioelectrochemical DNA detection technology was originally developed at the California Institute of Technology. The essence of the technology rests on specific detection of the electrical current generated by the reversible and continuous oxidation and reduction of labeled nucleic acid targets. The eSensor DNA detection technology exploits the electronic properties of nucleic acids while relying on the central principle of clinical molecular diagnostics, namely, hybridization. eSensor detection microchips contain numerous electronically active "pads," each decorated with specific DNA capture probes. The number of pads and specificity of probes on a chip can be varied depending on the applications of interest. As an electron donor, a ferrocene organometallic complex is covalently attached to a signal probe that is complementary to a region of the target of interest. The signal probes serve to label the target upon hybridization and are called AMBER (amperometric bioelectronic reporter) probes. The capture probes are attached to a gold microelectrode through phenylacetylene linkers that maintain the desired electrical contact between the probes and the surface of the gold electrode. Sample preparation, consisting of simple lysis of the cells, bacteria, or viruses of interest followed by denaturation and fragmentation, will not need to be augmented by significant purification. A complete test is performed as a sequence of events: $(i)$ introduction of the specimen, $(i i)$ a few moments of incubation for lysis and nucleic acid denaturation and fragmentation, (iii) several minutes of incubation for hybridization to occur, (iv) signal generation (initiated by pushing one button to generate the necessary voltage), and ( $v)$ display and read-out of result.

An improved method for producing microarrays that detect anthrax or smallpox in environmental samples has been developed at the Department of Energy's Pacific Northwest National Laboratory (PNNL; Richland, WA, USA) in conjunction with Washington State University (Pullman, WA, USA) (74,75). The microarrays consist of tiny probes placed on a glass plate. Each probe is sensitive to a specific pathogen marker, such as a DNA sequence encoding a toxin. Increased sensitivity is achieved by purification and concentration of bio-threat markers (such as whole cells, DNA, or proteins) and PCR amplification of nucleic acids prior to detection. The automated sample processing system, called the BattellePNNL Enhanced Biodetection Enabling Analyte Delivery System (BEADS) has been developed and used for purification and concentration of bio-threat markers. This system relies upon the manipulation of microparticle suspensions in a fluidic system and is well-suited for reversible sensing applications, automated serial assays, on-column or off-column detection, and biological or chemical separations/detection. The BEADS device can capture samples large enough to perform DNA testing in the field, automatically, 24 hours a day, and with no technician. Each bead is chemically designed to act like flypaper, isolating bacteria, spores, viruses, and their DNA from liquid samples derived from air, water, and dirt samples. The analytes are bound onto the surface of the beads, and the sample matrix materials that can interfere with PCR and detection are washed away. The analytes are then eluted from the beads and moved through a detector.
Results from testing in the field can be sent electronically to a remote location. With BEADS sample preparation systems as a front-end technology, detectors can be deployed as unattended bio-threat monitors in the field.

\section{CONCLUSION AND FUTURE AVENUES}

Sensitive and rapid detection of BWAs is being addressed with largely positive results because of major advances in PCR and microarray technology. Progress in microarray technology portends the application of hybridization-based approaches for detection of BWAs in the near future. The obvious advantages offered by the massively parallel form of analysis with microarrays include increased data per unit time and significant reduction in the time of analysis, sample volume required, and reagents consumed. These advantages are offset to some extent by the size and delicate nature of the current generation of system, limiting their use to laboratory settings.

Similarly, real-time PCR systems are playing an increasingly important role in health care, agriculture, and environmental monitoring. Military and civilian applications of these types of assays have clearly demonstrated ultrasensitive determination of microorganisms and viruses in a broad range of environments, including municipal water supplies, food products, or in plant, animal, or human tissues. In the meantime, however, considerable work remains to be done. One major technical challenge is to breach the detection barrier posed by complex environmental samples containing BWAs with sensitive detectors that can discriminate target cells, proteins, or nucleic acids. Field application of PCR remains limited in part because of its inherent complexity and the need for highly trained personnel for operation and interpretation of the results. While PCR assays are theoretically capable of detecting as little as one organism in a sample, this sensitivity also makes the tests susceptible to contamination that can cause false-positive results. In this respect, automation of detection processes for biological warfare agents remains one of the most important technical barriers for the deployment of PCR-based biodetectors in the field. The next generation of field detection devices should be fully automated devices with integrated sample preparation and biosensing elements, able to discriminate potential agents in a multianalyte environment, and report results autonomously. Full automation of assays and improved specimen-processing procedures can overcome many of the problems associated with first-generation tests, reducing human error, and increasing the accuracy of results. Continued progress in automated nucleic acid detection technologies will make real-time testing a reality under any environmental or biomedical application and permit more rapid and accurate monitoring of biological threat agents.

\section{ACKNOWLEDGMENTS}

This work was sponsored under a subcontract to the New Mexico Institute of Mining and Technology through the U.S. Air Force Research Laboratories Air Expeditionary Forces Technologies Division and General Atomics Systems 
Engineering Division, under contract USAFRL Contract F08630-01-C-0065. This article is part of a special journal section entitled "Rapid Detection and Identification of Infectious Disease Agents," edited by Leonard Peruski and Anne Harwood Peruski, Indiana University School of Medicine, Northwest Center, Gary, IN, USA.

\section{REFERENCES}

1.Spencer, R.C. 2003. Bacillus anthracis. J. Clin. Pathol. 56:182-187.

2.Greenfield, R.A., B.D. Lutz, M.M. Huycke, and M.S. Gilmore. 2002. Unconventional biological threats and the molecular biological response to biological threats. Am. J. Med. Sci. 323:350-357.

3.Beeching, N.J., D.A.B. Dance, A.R.O. Miller, and R.C. Spencer. 2002 Biological warfare and bioterrorism. Brit. Med. J. 324:336-339.

4.Deisingh, A.K. and M. Thompson. 2002. Detection of infectious and toxigenic bacteria. Analyst 127:567-581.

5.Walt, D. and D.R. Franz. 2000. Biological warfare detection. Anal. Chem. 72:739A-746A.

6.Iqbal, S.S., M.W. Mayo, J.G. Bruno, B.V. Bronk, C.A. Batt, and J.P. Chambers. 2000. A review of molecular recognition technologies for detection of biological threat agents. Biosens. Bioelectron. 15:549-578.

7.Norton, D.M. 2002. Polymerase chain reaction-based methods for detection of Listeria monocytogenes: toward real-time screening for food and environmental samples. J. AOAC Int. 85:505-515.

8.Min, J.H. and A. Baeumner. 2003. The micro-total analytical system for the detection of bacteria/viruses. J. Ind. Eng. Chem. 9:1-8.

9.Stenger, D.A., J.D. Andreadis, G.J. Vora, and J.J. Pancrazio. 2002. Potential applications of DNA microarrays in biodefense-related diagnostics. Curr. Opin. Biotech. 13:208-212.

10.Shoemaker, D.D. and P.S. Linsley. 2002. Recent developments in DNA microarrays. Curr. Opin. Microbiol. 5:334-337.

11.Rowe, C.A., L.M. Tender, M.J. Feldstein, J.P. Golden, S.B. Scruggs, B.D. MacCraith, J.J. Cras, and F.S. Ligler. 1999. Array biosensor for simultaneous identification of bacterial, viral, and protein analytes. Anal. Chem. 71:3846-3852.

12.McDonald, R., T. Cao, and R. Borschel. 2001. Multiplexing for the detection of multiple biowarfare agents shows promise in the field. Mil. Med. 166:237-239.

13.Lee, M.A., G. Brightwell, D. Leslie, H. Bird, and A. Hamilton. 1999. Fluorescent detection techniques for real-time multiplex strand specific detection of Bacillus anthracis using rapid PCR. J. Appl. Microbiol. 87: 218-223.

14.Uhl, J.R., C.A. Bell, L.M. Sloan, M.J. Espy, T.F. Smith, J.E. Rosenblatt, and F.R. Cockerill. 2002. Application of rapid-cycle real-time polymerase chain reaction for the detection of microbial pathogens: the Mayo-Roche rapid anthrax test. Mayo Clin. Proc. 77:673-680.

15.Reynolds, K.A., C.P. Gerba, M. Abbaszadegan, and I.L. Pepper. 2001 ICC/PCR detection of enteroviruses and hepatitis A virus in environmental samples. Can. J. Microbiol. 47:153-157.

16.Stratis-Cullum, D.N., G.D. Griffin, J. Mobley, A.A. Vass, and T. VoDinh. 2003. A miniature biochip system for detection of aerosolized Bacillus globigii spores. Anal. Chem. 75: 275-280.

17.Ivnitski, D., I. Abdel-Hamid, P. Atanasov, and E. Wilkins. 1999. Biosensors for detection of pathogenic bacteria. Biosens. Bioelectron. 14:599-624

18.Bell, C.A., J.R. Uhl, T.L. Hadfield, J.C. David, R.F. Meyer, T.F. Smith, and F.R. Cockerill. 2002. Detection of Bacillus anthracis DNA by LightCycler PCR. J. Clin. Microbiol. 40:2897-2902.

19.Ellerbrok, H., H. Nattermann, M. Ozel, L. Beutin, B. Appel, and G. Paul. 2002. Rapid and sensitive identification of pathogenic and apathogenic Bacillus anthracis by real-time PCR. FEMS Microbiol. Lett. 214: 51-59.

20.Hoffmaster, A.R., R.F. Meyer, M.D. Bowen, C.K. Marston, R.S. Weyant, G.A. Barnet, J.J. Sejvar, J.A. Jernigan, et al. 2002. Evaluation and validation of a real-time polymerase chain reaction assay for rapid identification of Bacillus anthracis. Emerg. Infect. Dis. 8:1178-1182.

21.Henchal, E.A., J.D. Teska, G.V. Ludwig, D.R. Shoemaker, and J.W. Ezzell. 2001. Current laboratory methods for biological threat agent iden- tification. Clin. Lab. Med. 21:661-663.

22.Paddle, B.M. 1996. Biosensors for chemical and biological agents of defense interest. Biosensor Bioelectron. 11:1079-1113.

23.Uithoven, K.A., J.C. Schmidt, and M.E. Ballman. 2000. Rapid identification of biological warfare agents using an instrument employing a light addressable potentiometric sensor and a flow-through immunofiltrationenzyme assay system. Biosensor Bioelectron. 14:761-770.

24.Lobenhofer, E.K., P.R. Bushel, C.A. Afshari, and H.K. Hamadeh. 2001. Progress in the application of DNA microarrays Environ. Health Persp. 109:881-891.

25.Jordan, J.A. 2000. Real-time detection of PCR products and microbiology. New Tech. Life Sci. 12:61-66.

26.Service, R.F. 2002. Analytical chemistry: new test could speed bioweapon detection. Science 295:1447.

27.Stix, G. 2002. The universal biosensor. A drug company tries to make a detector that can find nearly any biopathogen. Sci. Am. 287:37-39.

28.Hearps, A., Z. Zhang, and S. Alexandersen. 2002. Evaluation of the portable Cepheid SmartCycler real-time PCR machine for the rapid diagnosis of foot-and-mouth disease. Vet. Rec. 150:625-628.

29.Belanger, S.D., M. Boissinot, C. Menard, F.J. Picard, and M.G. Bergeron. 2002. Rapid detection of Shiga toxin-producing bacteria in feces by multiplex PCR with molecular beacons on the smart cycler. J. Clin. Microbiol. 40:1436-1440.

30.Belgrader, P., M. Okuzumi., F. Pourahmadi, D.A. Borkholder, and M.A. Northrup. 2000. A microfluidic cartridge to prepare spores for PCR analysis. Biosensor Bioelectron. 14:849-852.

31.Lee, L.G., K.J. Livak, B. Mullah, R.J. Graham, R.S. Vinayak, and T.M. Woudenberg. 1999. Seven-color, homogeneous detection of six PCR products. BioTechniques 27:342-349.

32.Swartzman, E.E., Miraglia, S.J., Mellentin-Michelotti, J., Evangelista, L., and P.M. Yuan. 1999. A homogeneous and multiplexed immunoassay for high-throughput screening using fluorometric microvolume assay technology. Anal. Biochem. 271:143-151

33.Schilling, E.A., A.E. Kamholz, and P. Yager. 2002. Cell lysis and protein extraction in a microfluidic device with detection by a fluorogenic enzyme assay. Anal. Chem. 74:1798-1804.

34.Schuch, R., D. Nelson, and V.A. Fischetti. 2002. A bacteriolytic agent that detects and kills Bacillus anthracis. Nature 418:884-889.

35.Belgrader, P., D. Hansford, G.T. Kovacs, K. Venkateswaran, R. J. Mariella, F. Milanovich, S. Nasarabadi, M. Okuzumi, et al. 1999. A minisonicator to rapidly disrupt bacterial spores for DNA analysis. Anal. Chem. 71:4232-4236

36.Belgrader, P., W. Benett, D. Hadley, J. Richards, P. Stratton, R. Mariella, and F. Milanovich. 1999. PCR detection of bacteria in seven minutes. Science 284:449-450

37.Belgrader, P., J.K. Smith, V.W. Weedn, and M.A. Northrup. 1998 Rapid PCR identity testing using a battery-powered miniature thermal cycler. J. Forensic Sci. 43:315-319.

38.Northrup, M.A., B. Benett, D. Hadley, P. Landre, S. Lehew, J. Richards, and P. Stratton. 1998. A miniature analytical instrument for nucleic acids based on micromachined silicon reaction chamber. Anal. Chem. 70:918-922.

39.Huang, J., F.J. DeGraves, D. Gao, P. Feng, T. Schlapp, and B. Kaltenboeck. 2001. Quantitative detection of Chlamydia spp by fluorescent PCR in the LightCycler. BioTechniques 30:150.

40.Makino, S.I., H.I. Cheun, M. Watarai, I. Uchida, and K. Takeshi. 2001. Detection of anthrax spores from the air by real-time PCR. Lett. Appl. Microbiol. 33:237-240.

41.Chapin, K. and T.L. Lauderdale. 1997. Evaluation of a rapid Air Thermal Cycler for detection of Mycobacterium tuberculosis. J. Clin. Microbiol. 35:2157-2159.

42.Jungkind, D. 2001. Automation of laboratory testing for infectious diseases using the polymerase chain reaction: our past, our present, our future. J. Clin. Virol. 20:1-6.

43.DiDomenico, N., H. Link, R. Knobel, T. Caratsch, W. Weschler, Z.G. Loewy, and M. Rosenstraus. 1996. COBAS AMPLICOR: Fully automated RNA and DNA amplification and detection system for routine diagnostic PCR. Clin. Chem. 42:1915-1923.

44.Benoit, V., A. Steel, M. Torres, Y.-Y. Yu, H. Yang, and J. Cooper. 2001 Evaluation of three-dimensional microchannel glass biochips for multiplexed nucleic acid fluorescence hybridization assays. Anal. Chem. 73: 2412-2420 
45.Schöning, M.J., A. Kurowski, M. Thust, P. Kordos, J.W. Schultze, and H. Lüth. 2000. Capacitive microsensors for biochemical sensing based on porous silicon technology. Sensor. Actuat. B-Chem. 64:59-64.

46.Liu, J., M. Enzelberger, and S. Quake. 2002. A nanoliter rotary device for polymerase chain reaction. Electrophoresis 23:1531-1536.

47.Peter, C., M. Meusel, F. Grawe, A. Katerkamp, K. Cammann, and T. Borchers. 2001. Optical DNA-sensor chip for real-time detection of hybridization events. Fresen. J. Anal. Chem. 371:120-127.

48.Taton, T.A., C.A. Mirkin, and R.L. Letsinger. 2000. Scanometric DNA array detection with nanoparticle probes. Science 289:1757-1760.

49.Reichert, J., A. Csaki, J.M. Kohler, and W. Fritzsche. 2000. Chipbased optical detection of DNA hybridization by means of nanobead labeling. Anal. Chem. 72:6025 -6029.

50.Lockhart, D.J. and E.A. Winzeler. 2000. Genomics, gene expression and DNA arrays. Nature 405:827-836.

51.Kricka, L.J. 2001. Microarrays, biochips and nanochips: personal laboratories for the 21st Century. Clin. Chem. Acta 307:219-223.

52.Boone, T.D., Z.H. Fan, H.H. Hooper, A.J. Ricco, H. Tan, and S.J. Williams. 2002. Plastic advances microfluidic devices. Anal. Chem. 74: 78A-86A.

53.Kopf-Sill, A.R. 2002. Success and challenges of lab-on-a-chip. Lab Chip $2: 42 \mathrm{~N}-47 \mathrm{~N}$

54.Chow, A.W. 2002. Lab-on-a-chip: opportunities for chemical engineering. AIChE J. 8:1590-1595.

55.Shimada, Y. 2002. Application of Affymetrix GeneChip system for plant hormone research. Plant Cell Physiol. 43:S2.

56.Merriman, B.L., Z. Chen, and S. Nelson. 2001. Improved quantitation of Affymetrix GeneChip microarray data. Am. J. Hum. Genet. 69:450.

57.Sanders, G.H.W. and A. Manz. 2000. Chip-based microsystems for genomic and proteomic analysis. Trends Anal. Chem. 19:304-318.

58.Shi, M.M. 2001. VistaArray technology: an integrated high throughput platform for drug discovery and personalized medicine. J. Clinic. Ligand Assay 24:145-148.

59.Nachamkin, I., N.J. Panaro, M. Li, H. Ung, P.K. Yuen, L.J. Kricka, P. Wilding. 2001. Agilent 2100 bioanalyzer for restriction fragment length polymorphism analysis of the Campylobacter jejuni flagellin gene. J. Clin. Microbiol. 39:754-757.

60.Lu, C.Y., D.J. Tso, T. Yang, Y.J. Jong, and Y.H. Wei. 2002. Detection of DNA mutations associated with mitochondrial diseases by Agilent 2100 bioanalyzer. Clin. Chim. Acta 318:97-105.

61.Birch, L., C.L. Archard, H.C. Parkes, and D.G. McDowell. 2001. Evaluation of LabChip ${ }^{\mathrm{TM}}$ technology for GMO analysis in food. Food Control 12:535-540.

62.Cunningham, D.D. 2001. Fluidics and sample handling in clinical chemical analysis. Anal. Chim. Acta 429:1-18.

63.Lichtenberg, J., N.F. de Rooij, and E. Verpoorte. 2002. Sample pretreatment on microfabricated devices. Talanta 56:233-266.

64.Righetti, P.G., C. Gelfi, and M.R. D'cunto. 2002. Recent progress in DNA analysis by capillary electrophoresis. Electrophoresis 23:13611374.

65.Haab, B.B. and R.A. Mathies. 1999. Single-molecule detection of DNA separations in microfabricated capillary electrophoresis chips employing focused molecular streams. Anal. Chem. 71:5137-5145.

66.Lagally, E.T., I. Medintz, and R.A. Mathies. 2001. Single-molecule DNA amplification and analysis in an integrated microfluidic device. Anal. Chem. 73:565-570.

67.Pethig, R., J.P.H. Burt, A. Parton, N. Rizvi, M.S. Talary, and J.A. Tame. 1998. Development of biofactory-on-a-chip technology using excimer laser micromachining. J. Micromech. Microeng. 8:57-63.

68.Steel, A., M. Torres, J. Hartwell, Y-Y. Yu, N. Ting, G. Hoke, and H. Yang. 2000. The Flow-Thru Chip ${ }^{\mathrm{TM}}$ : a three-dimensional biochip platform, p. 87-117. In M. Schena (Ed.), Microarray Biochip Technology. Eaton Publishing, Natick, MA.

69.Cheek, B. J., A. B. Steel, M. P. Torres, Y-Y. Yu, and H Yang. 2001. Chemiluminescence detection for hybridization assays on the Flow-Thru Chip, a three-dimensional microchannel biochip. Anal. Chem. 73:57775783 .

70.Yang, J.M., J.L. Bell, Y. Huang, M. Tirado, D. Thomas, A.H. Forster, R.W. Haigis, P.D. Swanson, et al. 2002. An integrated, stacked microlaboratory for biological agent detection with DNA and immunoassays. Biosensor. Bioelectron. 17:605-618.

71.Forster, A.H., M. Krihak, P.D. Swanson, T.C. Young, and D.E. Ackley.
2001. A laminated, flex structure for electronic transport and hybridization of DNA. Biosensor. Bioelectron. 16:187-194.

72.Liu, Y.B., W.M. Shi, G.E. Park, A. Asatourians, Y. Chen, D. Farkas, and B. Coty. 2002. Electrochemical detection on eSensor ${ }^{\mathrm{TM}}$ DNA chips for genotyping mutations in the cystic fibrosis transmembrane conductance regulator gene. Clin. Chem. 48:A179.

73.Shi, W.M., M.R. Reed, Y.B. Liu, L.N. Cheung, R.J. Wu, A. Asatourians, G.E. Park, and B. Coty. 2002. Cystic fibrosis carrier screening using electrochemical detection on the eSensor DNA detection platform. Clin. Chem. 48:2090.

74.Call, D. R., D. P. Chandler, and F. J. Brockman. 2001. Fabrication of DNA microarrays using unmodified oligomer probes. BioTechniques 30 : 368-379.

75.Chandler, D.P., J. Brown, D.R. Call, S. Wunschel, J.W. Grate, D.A. Holman, L. Olson, M.S. Stottlemyre, and C.J. Bruckner-Lea. 2001 Automated immunomagnetic separation and microarray detection of E. coli O157:H7 from poultry carcass rinse. Int. J. Food Microbiol. 70: 143-154.

Address correspondence to:

Dmitri Ivnitski

Institute for Engineering Research and

Applications

New Mexico Institute of Mining and Technology

901 University Blvd. SE

Albuquerque, NM 87106-4339, USA

e-mail:vinitski@nmt.edu 